\title{
Dimensional Analysis of Thrusting by Electromagnetic Inertia
}

\author{
Mario J. Pinheiro ${ }^{1}$ \\ Department of Physics, Instituto Superior Tecnico - IST, Universidade de Lisboa - UL, Av. Rovisco Pais, \\ 83 1049-001 Lisboa, Portuga 国
}

( $\Omega$ Dated: 9 February 2015)

We investigate the so called Biefeld-Brown effect in terms of dimensional analysis and in the frame of classical electrodynamics.

PACS numbers: 03.50.De,72.30.+q,77.22.-d,84.37.+q,84.30.Jc

Keywords: Classical electromagnetism,Maxwell equations;High-frequency effect;plasma effects;Dielectric properties of solids and liquids;Electric variables measurements;Power electronics;power supply circuits

\section{INTRODUCTION}

The so called Biefeld-Brown effect is hypothetically a means to convert electrostatic energy into a propulsive force, even in a vacuum medium. It was discovered by Thomas Townsend Brown and Dr. Paul Alfred Biefeld. In 1921 Brown discovered this effect when experimenting with a Coolidge X-ray tube. The tube consisted of two asymmetrical electrodes separated by a dielectric in a vacuum environment. When connected to a high-voltage source a force acted on the tube, forcing its motion in the direction of the positive electrode $\mathrm{e}^{\underline{1}}$. In case this effect is real the associated potential for propulsion and as a source of energy is enormous since no expenditure of fuel is necessary, the all process remaining (most probably) nevertheless connected to action-reaction type of momentum-transfer. Tests made at NASA report thrust produced for various voltages, polarities and ground configurations, leading to design asymmetrical capacitors for propulsion $\underline{3}$.

In 1893 Heaviside $\frac{12}{2}$ proposed the separation of gravitation int electric and magnetic components.

Some Authors use the weak-field approximation to modify the equations of the general theory of gravitation in a structurally similar kind of equations as the Maxwell equations of the electromagnetism 16 . We can find also in Iwanaga ${ }^{6}$ a review of some field propulsion methods based on general relativity theory. Nevertheless, so far proposed concepts to control gravity for possible use on space propulsion don't lead to no breakthrough ${ }^{7}$.

Another interpretation relies on the standard atmospheric electrodynamic model of the global electric circuit. The global electric circuit is composed by the spherical conductor of the earth, the spherical conductor of the ionosphere. Thunderstorms act as charge generators, charging negatively the earth surface and charging the ionosphere positive. Statistically the electric field strength reaches maximums (at 18:00 UTC) and minimums (at 04:00 UTC) everywhere on earth according to

\footnotetext{
a) Electronic mail: mpinheiro@tecnico.ulisboa.pt We acknowledge financial support from Fundação para a Ciência e Tecnologia through award SFRH/BSAB/1420/2014
}

UTC time and not the local time. This was experimentally verified with data taken on board the ship Carnegie and that is why is characteristic diurnal curve is known as the "Carnegie curve". The self-potential measured by Brown with a capacitor and a rock sample between the electrodes acting as a dielectric, exposed to the Earth background electric field apparently shows several parallels. Stephenson ${ }^{\underline{8}}$ suggested that the Biefeld-Brown effect is a secondary electrostatic effect related to the global electric field. This interpretation precludes any relationship with the electrogravitic nature of the effect.

Through a lagrangian formulation Feigel $\underline{18}$ shown that a body receives a recoil momentum from the vacuum equal to the Minkowski's momentum, opening the possibility of contribution of vacuum to the motion of dielectric liquids in crossed electric and magnetic fields. Exploring different possibilities, Maclay and Forward 31 proposed a mechanism to propel a spacecraft based on the Casimir effect, in which electromagnetic radiation is emitted when an uncharged mirror is properly accelerated in vacuum.

Following the claims from Podkletnov $\underline{45}$ of the possible connection between gravity and electro-magnetic effects on type II, YBCO superconductors, experimental research has been done to verify it without success $\underline{46}$.

Loder ${ }^{10}$ gives an overall account of technologies applications for the $21^{\text {st }}$ century. $\mathrm{In}^{\underline{9}}$ it is discussed the possibility of engineering the zero-point field and polarizable vacuum for propellantless propulsion and an idealized system composed of two parallel semiconducting boundaries separated by an empty gap variable width can, under appropriate transformation, generate work ${ }^{11}$.

\section{WHAT IS COMMON KNOWLEDGE ABOUT THE BIEFELD-BROWN EFFECT}

According to experiments done by Thomas Townsend Brown which lead to several patents registered in US and Great Britain $\underline{1}^{1}, \underline{2}$, the propulsive effect is dependent on the following factors:

1. the surface area of the electrodes, $S$;

2. the voltage differential between the electrodes, $V$; 
TABLE I. Dimensions of the relevant physical variables Permittivity of vacuum $\left[\varepsilon_{0}\right]=M L T^{-2} Q^{-1}$ Electric scalar potential $[V]=M L^{2} T^{-2} Q^{-1}$ Force

$[F]=\quad M L T^{-2}$

3. the distance between the electrodes, $d$;

4. the kind of material used between the masses, $\rho_{m}$;

5. the dielectric permittivity of the material placed between the electrodes, $\epsilon_{r}$.

This information suggests that the force is directly dependent on the system capacitance and the total charge developed, $Q=C V$, as the dimensional analysis of Section 2 clearly shows.

\section{A DIMENSIONAL ANALYSIS}

We now apply the Buckingham's Pi theorem in order to obtain the expected relationship between the operational variables related to this problem, in particular, according to the Biefeld-Brown investigations. In Table I we present the electromagnetic magnitudes with relevance to our problem.

Through a criterious application of the Buckingham's Pi theorem we conclude that

$$
F=G \varepsilon \frac{V^{2}}{d} \mathcal{F}\left(\frac{S}{d}\right)
$$

where $G$ is a constant to be determined by means of a theoretical explanation or experimental data, and $\mathcal{F}(S / d)$ is a function dependent on the ratio $S / d$.

\section{INTERACTION WITH THE VACUUM}

Although recently Newton's third law of motion was at $\operatorname{stack}^{15}$ it is likely action and reaction always occurs by pairs and $\mathbf{F}=-\mathbf{F}^{\prime}$ holds.

According to the Maxwell's theorem, the resultant of $\mathbf{K}$ forces applied to bodies situated within a closed surface $S$ is given by the integral over the surface $S$ of the Maxwell stresses:

$$
\int \mathbf{T}(n) d S=\int \mathbf{f} d \Omega=\mathbf{K} .
$$

Here, $\mathbf{f}$ is the ponderomotive forces density and $d \Omega$ is the volume element. The vector $\mathbf{T}(n)$ under the integral in the left-hand side (lhs) of the equation is the tension force acting on a surface element $d S$, with a normal $\mathbf{n}$ directed toward the exterior. In cartesian coordinates, each component of $\mathbf{T}(n)$ is defined by

$$
T_{x}(n)=t_{x x} \cos (n, x)+t_{x y} \cos (n, y)+t_{x z} \cos (n, z),
$$

with similar expressions for $T_{y}$ and $T_{z}$. The 4dimensional momentum-energy tensor is a generalization of the 3-dimensional stress tensor $T_{l m}$. If electric charges are inside a conducting body in vacuum, in presence of electric $E$ and magnetic $H$ fields, then Eq. 2 must be modified to the form

$$
\int \mathbf{T}(n) d S-\mathbf{K}=\int \frac{1}{4 \pi c}\left(\frac{\partial[\mathbf{E} \times \mathbf{H}]}{\partial t}\right) d \Omega .
$$

In the right-hand side of the above equation it now appears the temporal derivative of $\mathbf{G}=\int \mathbf{g} d \Omega$, the electromagnetic momentum of the field in the entire volume contained by the surface $S$ (with $\mathrm{g}$ its momentum density).

In the case the surface $S$ is filled with a homogeneous medium without true charges, Abraham proposed to write instead

$$
\int \mathbf{T}(n) d S=\frac{\partial}{\partial t} \int \frac{\varepsilon \mu}{4 \pi c}[\mathbf{E} \times \mathbf{H}] d \Omega,
$$

with $\varepsilon$ and $\mu$ the dielectric constant of the medium and its magnetic permeability.

Eq. 5 can be written on the form of a general conservation law

$$
\frac{\partial \sigma_{\alpha \beta}}{\partial x_{\beta}}-\frac{\partial g_{\alpha}}{\partial t}=f_{\alpha}
$$

where $\alpha=1,2,3$.

This equation can be reduced to the form

$$
\frac{\partial \sigma_{\alpha \beta}}{\partial x_{\beta}}=f_{\alpha}^{L}+\frac{1}{4 \pi c} \frac{\partial}{\partial t}[\mathbf{D} \times \mathbf{B}]_{\alpha}+f_{m, \alpha}^{\prime} .
$$

Here, $f_{m}^{\prime}$ is the force acting in the medium ${ }^{20}, \mathbf{f}^{L}=\rho_{e} \mathbf{E}+$ $\frac{1}{c}[\mathbf{j} \times \mathbf{B}]$ is the Lorentz force density with $\rho_{e}$ denoting the charge density and $\mathbf{j}$ the current density.

Of course, the field and the the medium (or the matter) form together a closed system and it is usual to catch the momentum conservation law in the general form $\underline{42,44}$

$$
\frac{\partial\left(T_{\alpha \beta}^{\text {Field }}+T_{\alpha \beta}^{\text {Matter }}\right)}{\partial x_{\beta}}=0 .
$$

The general relation between Minkowski and Abraham momentum, free of any particular assumption, holding particularly for a moving medium, is given by

$$
\mathbf{P}^{M}=\mathbf{P}^{A}+\int \mathbf{f}^{A} d t d V
$$

For clearness, we shall distinguish between the parts of a system, the body carrying currents and the currents themselves (the structure for short), the fields and the vacuum. 
The impulse transmitted to the structure is just

$$
\mathbf{P}^{K}=\int \mathbf{f}^{A} d t d V=\mathbf{P}^{M}-\mathbf{P}^{A},
$$

where $\mathbf{f}^{A}$ is the Abraham's force density:

$$
\mathbf{f}^{A}=\frac{\varepsilon_{r} \mu_{r}-1}{4 \pi c} \frac{\partial[\mathbf{E} \times \mathbf{H}]}{\partial t} .
$$

This is in agreement with experimental data $\underline{\underline{43}}$ and was proposed by others $\underline{47,48}$. As this force is acting over the medium, it is expected nonlinearities related to the behavior of the dielectric to different applied frequencies, temperature,pressure, and large amplitudes of the electric field when a pure dielectric response of the matter is no longer proportional to the electric field (see Ref. $\underline{49}$ on this topic).

The momentum conservation law can be rewritten as ( $\operatorname{see} \stackrel{20}{\underline{n}})$

$$
\frac{\partial \sigma_{\alpha \beta}}{\partial x_{\beta}}=f_{\alpha}^{L}+\frac{1}{4 \pi c} \frac{\partial}{\partial t}[\mathbf{D} \times \mathbf{B}]_{\alpha}+f_{m, \alpha}^{\prime},
$$

with $f_{m}^{\prime}$ denoting the force acting on the medium. The second term in the lhs of above equation could possible be called vacuum-interactance term $\underline{21}$ - in fact, Minkowski term. Already according to an interpretation of Einstein and Laub $\stackrel{22}{2}$, the integration of above equation over all space, the derivative over stress tensor gives a null integral and the Lorentz forces summed over all the universe must be balanced by the quantity $\int_{\infty} \varepsilon_{0} \mu_{0} \frac{\partial[\mathbf{E} \times \mathbf{H}]}{\partial t} d V$ in order Newton's third law be preserved.

As is well known, Maxwell's classical theory introduces the idea of a real vacuum medium. After being considered useless by Einstein's special theory of relativity, the ether (actually replaced by the term vacuum or physical vacuum) was rehabilitated by Einstein in $1920 \underline{23}$. In fact, general theory of relativity describes space with physical properties by means of ten functions $g_{\mu \nu}$ (see also $\stackrel{32}{ }$ ). According to Einstein,

The ether of general relativity is a medium that by itself is devoid of all mechanical and kinematic properties but at the same time determines mechanical (and electromagnetic) processes.

Dirac felt the necessity to introduce the idea of ether in quantum mechanics 28 . In fact, according to quantum field theory, particles can condense in vacuum giving rise to space-time dependent macroscopic objects, for example, of ferromagnetic type. Besides, stochastic electrodynamics shown that the vacuum contains measurable energy called zero-point energy (ZPE) described as a turbulent sea of randomly fluctuating electromagnetic field. Quite interestingly, it was recently shown that the interaction of atoms with ZPF guarantees the stability of matter and, in particular, the energy radiated by an accelerated electron in circular motion is balanced by the energy absorbed from the ZPF 34

Graham and Lahoz made three important experiments ${ }^{24}-26$. While the first experiment provided an experimental observation of Abraham force in a dielectric, the second one provided a measurement of a reaction force which appear in magnetite. The third one provided the first evidence of free electromagnetic angular momentum created by quasistatic and independent electromagnetic fields $E$ and $B$ in the vacuum 27 . Whereas the referred paper by Lahoz provided experimental evidence for Abraham force at low frequency fields, it still remains to gather evidence of its validity at higher frequency domain, although some methods are presently outlined $\underline{39}$.

All this is known since a long time and we only try to put more clear the theoretical framework, that only needs to be experimentally tested for proof of principles.

In view of the above, we will write the ponderomotive force density acting on the composite body of arbitrarily large mass (formed by the current configuration and its supporting structure) in the form

$$
\rho \frac{d \mathbf{V}}{d t}=\rho_{c} \mathbf{E}+[\mathbf{J} \times \mathbf{B}]+\nabla \cdot \mathbf{T}+\frac{\partial}{\partial t}\left(\varepsilon_{0} \mu_{0}[\mathbf{E} \times \mathbf{H}]\right) .
$$

Hence, the composite body is acted on by Minkowski force in such a way that

$$
M \mathbf{V}=-\mathbf{G}^{M}+\mathbf{G}^{A} .
$$

The Minkowski momentum is transferred only to the field in the structure and not to the structure and the field in the medium $20,26,33$.

It seems that generally in nature, locomotory propulsion by oscillating flukes or wings are characterized by the periodic shedding of wake vortices (the so called Von Karman streets) inducing jet flows carrying momentum and thus the body will experience a reaction force the

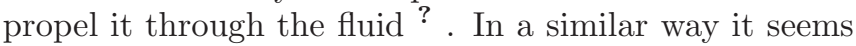
to occur with Minkowski force, which is vortex formed in ether and inducing a reaction force on the body which propels it in ether.

\section{PONDEROMOTIVE FORCE VIA VECTOR POTENTIAL}

Difficulties related to the Minkowski-Abraham controversy maybe avoided formulating the problem in term of canonical momentum, instead of ponderomotive forces. Canonical momentum allows a clear cut view of phenomena. In this theoretical frame there is no violation of action-reaction law. In fact, the massless propulsion is achieved obtaining mechanical momentum through electromagnetic momentum exchanged with the medium.

According to Trammel $\stackrel{35}{ }$, the total momentum acting over the composite body in an inertial frame can be also 
written in the form

$$
\mathbf{P}=\rho \mathbf{V}+\sum_{i} \rho_{c, i} \mathbf{v}_{i}+\sum_{i} \rho_{c, i} \mathbf{A}_{i} .
$$

This is supposed to apply to a structure and currentcarrying wires integrated inside the structure. It is assumed to exist a current density $\mathbf{J}=\sum_{i} \rho_{c, i} \mathbf{v}_{i}$. To simplify, we will assume that in the structure inertial frame the current undergoes arbitrary small acceleration, i.e., the current is quasistationary. Also, we discard the effect of the motion on the electromagnetic field.

We will assume a very simple geometry with two electrodes below and above a dielectric cylinder (e.g, baryum titanate). The leakage current will flow along z-axis and, consequently, so do the potential vector $\mathbf{A}=A_{z} \mathbf{u}_{z}$. Hence, the propulsive force will be acting along axis Oz:

$$
\mathbf{F}_{z}=M \frac{d V_{z}}{d t}=-Q \frac{d A_{z}}{d t}
$$

where $M$ (an arbitrary large mass) and $V_{z}$ are the mass of the composite body and its velocity along $z$-axis. The electromagnetic part of momentum acting over the charge $Q$ developed on electrodes making part of the structure of mass $M$ is $Q A_{z}$. $A_{z}$ is the vector potential component along $\mathrm{Oz}$, given by:

$$
A_{z}(r)=\frac{\mu_{0} J_{z}}{4} r^{2}
$$

It is independent of z-coordinate, depending only on the radial position. We denote by $J_{z}$ is the current density, $R$ the electrodes radius (by default, the lower smaller radius) and $d$ the capacitors width. The total charge $Q$ present on each electrode is such that $Q=C\left(V_{1}-V_{2}\right)$.

After integration over the charged electrode, the final expression for the force is readily obtained

$$
F_{z}=-\frac{1}{8 c_{0}^{2}} \varepsilon_{r} \omega I \frac{R^{2}}{d}\left(V_{1}-V_{2}\right) .
$$

Our result working along a second line is consistent with Hector's 19 finding, who obtained an analogous expression to Eq. 18. In fact, Eq. 18 is consistent with the dimensional analysis done using Buckingham' Pi theorem, since a dependency on energy retained between electrodes. If we restrain ourselves to simple considerations, and as long as the leakage current is given as $I \approx \Delta V / \mathcal{R}$, with $\mathcal{R}$ representing now the electrical resistance of the dielectric cylinder, the force is approximately given by

$$
F_{z} \approx \frac{10^{-7}}{2} \frac{C \omega}{\mathcal{R}} \Delta V^{2} .
$$

Eq. 18 also is consistent with Eq. 11 obtained through dimensional analysis, but now we have determined explicitly the form of function $\mathcal{F}(S / d)$.

\section{CONCLUSION}

We investigate the so called Biefeld-Brown effect, or similar devices operating on electromagnetic forces, in terms of dimensional analysis and in the frame of classical electrodynamics. In particular, we obtained explicitly the function dependent form $\mathcal{F}$. This result can be helpful when addressing electromagnetic propulsion devices.

${ }^{1}$ T. T. Brown, "Electrokinetic Apparatus", US Patent 2,949,550 (1960); "Electrokinetic Apparatus", US Patent 3,187,206 (1965);

"A method of and an apparatus or machine for producing force or motion", GB Patent 300,311 (1928)

${ }^{2}$ T. T. Brown, Science \& Invention Magazine (1929)

${ }^{3}$ Francis X. Canning, Cory Melcher and Edwin Winet, "Asymetrical capacitors for propulsion", NASA/CR-2004-213312

${ }^{4}$ T. B. Bahder and C. Fazi, "Force on an asymmetric capacitor", Final report, Aug.Dec. 2002, ARL-TR-3005, NTIS Number ADA416740

${ }^{5}$ R. L. Talley, "Twenty-First Century Propulsion Concept", PLTR-91-3009 (1991)

${ }^{6}$ N. Iwanaga, "Reviews of Some field propulsion methods from the general relativistic standpoint", Space Technology and Applications International Forum, CP458, Ed. Mohamed S. El-Genk (1999)

${ }^{7}$ M. Tajmar and O. Bertolami, Los Alamos archives physics/0412176 O. Bertolami, Report ESA CR(P)4365 (2002)

${ }^{8}$ G. V. Stephenson, "The Biefeld-Brown Effect and the Global Electric Circuit", preliminary -10/25/01 peer review copy, Seculine consulting, Bellevue, WA 98006 (2001)

${ }^{9}$ H.E.Puthoff, S.R.Little and M.Ibison, JBIS 55 137-144 (2002)

${ }^{10}$ Theodore C. Loder, Paper number AIAA-2002-1131

${ }^{11}$ F. Pinto, Phys. Rev. 60 (21) 14740-14755 (1999)

${ }^{12} \mathrm{O}$. Heaviside, A graviatitional and electromagnetic analogy, The Electrician 31 pp. 281-282 and 359 (1983)

${ }^{13}$ Robert L. Forward, Am. J. Phys. 31 (3) 166-170 (1963)

${ }^{14}$ Doyle R. Buehler, Journal of Space Mixing 2 1-22 (2004)

${ }^{15}$ Patrick Cornille, Can. J. Phys. 73 619-625 (1995)

${ }^{16}$ M. Tajmar and C. J. de Matos, Journal of Theoretics 3 (1) (2001)

${ }^{17}$ R. L. Talley, Twenty first century propulsion concept, Veritay Technology, May 1991

18 A. Feigel, Phys. Rev. Lett. 92(2) 020404-1 (2004)

${ }^{19}$ Hector H. Brito, Acta Astronautica 54 pp. 547-558 (2004)

${ }^{20}$ V. L. Ginzburg and V. A. Ugarov, Sov. Phys. Usp. 19(1), 94-101 (1976)

${ }^{21}$ Blair M. Clevelance, Electric Spacecraft 24 6-16 (1996)

${ }^{22}$ A. Eintein and J. Laub, Annls. Phys. (Leipzig) 26 541-550 (1908)

${ }^{23}$ A. Einstein, Aether und Relativitaetstheorie (Springer, Berlin, 1920)

${ }^{24}$ G. B. Walker and D. G. Lahoz, Nature 253 339-340 (1975)

${ }^{25}$ D. G. Lahoz and G. M. Graham, Phys. Rev. Lett. 42 (17) 1137$1140(1979)$

${ }^{26}$ G. M. Graham and D. G. Lahoz, Nature 285 (5761) 154-155 (1980)

${ }^{27}$ In 26, ("According to Maxwell-Poynting ideas, the last (Minkowski's) term in [our Eq.1] can be interpreted as a local reaction force acting on charges and currents when the vacuum surrounding them is loaded with electromagnetic momentum."

${ }^{28}$ P. Dirac, Nature 168906 (1951)

${ }^{29}$ W. Shockley and R.P.James, Phys. Rev. Lett. 18 (20) 876 (1967)

${ }^{30}$ Anders Hedenström, TRENDS in Ecology and Evolution, 19(5) 217-219 (2004)

${ }^{31} \mathrm{G}$. Jordan Maclay and Robert L. Forward, Foundations of Physics 34(3) 477 (2004)

32 V. L. Ginzburg and V. P. Frolov, Sov. Phys. Usp. 30 (12) 1073 (1987)

${ }^{33}$ D. V. Skobel'tsyn, Sov. Phys.-Usp. 16 (3), 381 (1974)

${ }^{34}$ M. Kozlowski and J. Marciak-Kozlowska, ?? 
${ }^{35}$ G. T. Trammel, Phys. Rev. 134(5B) B1183-B1184 (1964)

${ }^{36}$ T. T. Taylor, Phys. Rev. 137 (2B) B467-B471 (1965)

${ }^{37}$ Max Abraham, Rend. Circ. Matem. Palermo, t. XXVIII (2 ${ }^{\circ}$ sem. 1909)

${ }^{38}$ Max Abraham, Rend. Circ. Matem. Palermo, t. XXX (2o sem. 1910)

${ }^{39} \mathrm{~S}$. Antoci and L. Mihich EPJdirect D1, 1-11 (1998)

${ }^{40}$ Y. Aharonov and D. Bohm, Phys. Rev. 115485 (1959)

${ }^{41}$ Takaaki Musha, "Experiment on the TTBrown effect conducted by T. Musha", in Review of some field propulsion methods from the general relativistic standpoint Ed. by Iwanaga Noriki (??)
${ }^{42}$ Walter Thirring, Dynamical systems and field theories, (Springer, 1927) 3rd ed.

${ }^{43}$ R. V. Jones and J. C. S. Richard, Proc. R. Soc. A 221481 (1954)

${ }^{44}$ L. D. Landau and E. M. Lifshitz, Fluid Mechanics (Pergamon, Oxford, 1987), 2nd ed., Secs. 133 and 134

${ }^{45}$ E. Podkletnov and R. Niemen, Physica C, 203 441-444 (1992)

${ }^{46}$ Glen A. Robertson, and Ron Litchford, Bryan Thompson, Randall Peters, AIAA-2001-3364

${ }^{47}$ James P. Gordon, Phys. Rev. A 8 (1) 14-21 (1973)

${ }^{48}$ Frank R. Tangherlini, Phys. Rev. A 12 (1) 139-147 (1975)

${ }^{49}$ Ulrich Böttger, Polar Oxides: Properties, Characterizing, and Imaging, Edited by R. Waser, U. Böttger, and S. Tiedke, (WILEY-VCH Verlag GmbH \& Co. KGaA, Weinheim, 2005) 